Annals of Pure and Applied Mathematics

Vol. 15, No. 2, 2017, 295-304

ISSN: 2279-087X (P), 2279-0888(online)

Published on 11 December 2017

www.researchmathsci.org

DOI: http://dx.doi.org/10.22457/apam.v15n2a16

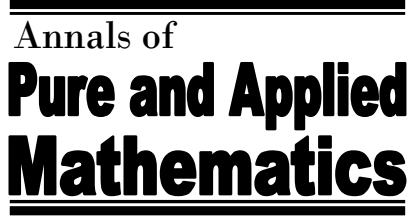

\title{
The Fermat S-Prime Meet Matrices and Reciprocal Fermat S-Prime Meet Matrices on Posets
}

N.Elumalai ${ }^{1}$ and R.Anuradha ${ }^{2}$

${ }^{1}$ Department of Mathematics, A.V.C College (Autonomous)

Mannampandal - 609305, Mayiladuthurai, India.

${ }^{1}$ E-mail:nelumalai@ rediffmail.com; ${ }^{2}$ Email: anuakshadha@ gmail.com

Received 25 November 2017; accepted 10 December 2017

Abstract. We consider fermat S-prime meet matrices and reciprocal fermat S-prime meet matrices on posets as an abstract generalization of fermat S-prime greatest common divisor (fermat S-prime GCD) matrices. Some of the most important properties of fermat S-prime GCD matrices are presented in terms of S-prime meet matrices.

Keywords: S-prime meet, reciprocal S-prime, fermat S-prime, reciprocal fermat $\mathrm{S}$-prime, fermat numbers

AMS Mathematics Subject Classification (2010): 06A06, 06A11

\section{Introduction}

Let $S=\left\{\mathrm{x}_{1}, \mathrm{x}_{2}, \ldots, \mathrm{x}_{\mathrm{n}}\right\}$ be a set of distinct positive integer, and let $\mathrm{f}$ be an arithmetical function. Then $n \times n$ matrix $(S)$ whose $\mathrm{i}, \mathrm{j}$-entry is the greatest common divisor $\left(\mathrm{x}_{\mathrm{i}}, \mathrm{x}_{\mathrm{j}}\right)$ of $x_{i}$ and $x_{j}$ is called the GCD matrix on $S[3,5,8]$.

In 1876, H. J. S. Smith [12] showed that the determinant of the GCD matrix defined on $S=\{1,2, \ldots . n\}$ (Smith's determinant) is equal to $\varphi(1) \varphi(2) \ldots \varphi(n)$, where $\varphi$ is Euler's totient function.

The set $\mathrm{S}$ is said to be factor-closed if it contains every divisor of any element of $\mathrm{S}$, and the set $\mathrm{S}$ is said to be GCD-closed if it contains the greatest common divisor of any two elements of $S[8]$.

The GCD matrix with respect to $\mathrm{f}$ is

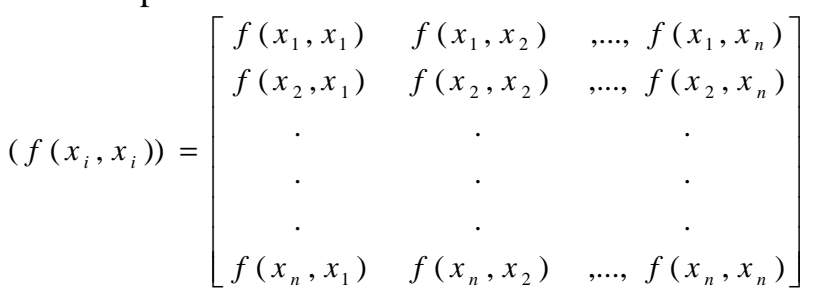

and $\operatorname{det}\left[\mathrm{f}\left(\mathrm{x}_{\mathrm{i}}, \mathrm{x}_{\mathrm{j}}\right)\right]=\prod_{k=1}^{n}(f * \mu)\left(x_{k}\right)$

In 1960, Carlitz [9], gave a new form of gcd-matrices and determinant value, $[\mathrm{f}(\mathrm{i}, \mathrm{j})]_{\mathrm{n}}=\mathrm{C}(\operatorname{diag}(\mathrm{g}(1), \ldots, \mathrm{g}(\mathrm{n}))) \mathrm{C}^{\mathrm{T}} \quad$ where $\mathrm{C}=\left(\mathrm{C}_{\mathrm{ij}}\right)_{\mathrm{nxn}}$; 
N.Elumalai and R.Anuradha

$C_{i j}=\left\{\begin{array}{l}1 \text { if } j \mid i \\ 0 \text { if } j \mid i\end{array} \quad\right.$ and $D=\left(d_{i j}\right)$ diagonal matrix where $d_{i j}=\left\{\begin{array}{lll}g(i) & \text { if } & i=j \\ 0 & \text { if } & i \neq j\end{array}\right.$

$\therefore \operatorname{det}[\mathrm{f}(\mathrm{i}, \mathrm{j})]_{\mathrm{nxn}}=\mathrm{g}(1) \cdot \mathrm{g}(2) \ldots \mathrm{g}(\mathrm{n})$

In 1992, Beslin and Ligh [4] generalized in this results on GCD matrices by showing that the determinant of the GCD Matrix on a GCD closed set $\mathrm{S}=\left\{\mathrm{x}_{1}, \mathrm{x}_{2}, \mathrm{x}_{3}, \ldots, \mathrm{x}_{\mathrm{n}}\right\}$ is the product $\prod_{k=1}^{n}\left(\alpha_{k}\right)$ Where $\alpha_{k}=\sum_{\begin{array}{l}d / x_{k} \\ d t x_{t} \\ x_{t}<x_{k}\end{array}} \Phi(d)$

2. Structure of fermat $S$-prime meet and reciprocal fermat S-prime meet matrices Definition 2.1. Let $(P, \prec)=\left(Z^{+}, \mid\right)$be a finite poset. We call $P$ be a meet - semi lattice if for any $\mathrm{x}, \mathrm{y} \in \mathrm{P}$ there exist a unique $\mathrm{z} \in \mathrm{P}$. such that (i) $\mathrm{z} \leq \mathrm{x}$ and $\mathrm{z} \leq \mathrm{y}$ and (ii) If $\mathrm{w}$ $\leq \mathrm{x}$ and $\mathrm{w} \leq \mathrm{y}$ for some $\mathrm{w} \in \mathrm{P}$.then $\mathrm{w} \leq \mathrm{z}$. In such a case $\mathrm{z}$ is called the meet of $\mathrm{x}$ and $\mathrm{y}$ is denoted by $x \wedge y$.

Definition 2.2. Let $\mathrm{S}$ be a subset of subset of $\mathrm{P}$.we call $\mathrm{S}$ be a lower- closed if for every $x, y \in P$ and $x \in S$ and $y \leq S$.we have $y \in S$.

Definition 2.3. Let $S$ be a subset of $P$ then $S$ is said to be meet-closed if for every $x, y \in$ $\mathrm{S}$ we have $x \wedge y \in \mathrm{S}$.

Definition 2.4. Let $\mathrm{x}$ and $\mathrm{y}$ be two elements of the poset $\mathrm{P}$ and $\mu$ is the Mobius function of the $\operatorname{poset}(S, \prec)$ then

$$
\mu(x, y)=\left\{\begin{array}{ccc}
0 & \text { if } & x \neq y \\
1 & \text { if } & x=y \\
-\Sigma \mu(x, z) & & \text { otherwise }
\end{array}\right\}
$$

Definition 2.5. Let $S=\left\{x_{1}, x_{2}, x_{3}, \ldots, x_{n}\right\}$ and $T=\left\{y_{1}, y_{2}, y_{3}, \ldots, y_{n}\right\}$ be any two subsets of P.Define the incidence matrix whose $\mathrm{i}, \mathrm{j}$ entry is 1 if $y_{j} \leq x_{i}$ and 0 otherwise, namely

$$
\mathrm{E}(\mathrm{S}, \mathrm{T})=\left(e_{i j}\right)_{n \times m}=\left\{\begin{array}{lll}
1 & ; & y_{j} \leq x_{i} \\
0 & ; & \text { otherwise }
\end{array}\right.
$$

Example 2.6. We consider $S=\{5,9,13\}, T=\{9,17,21\}$ are the $S$-prime number subsets.

Then the incidence matrix of $(\mathrm{S}, \mathrm{T})$ is

$$
E(S, T)=\left(e_{i j}\right)=\left[\begin{array}{lll}
0 & 0 & 0 \\
1 & 0 & 0 \\
1 & 0 & 0
\end{array}\right]
$$

Definition 2.7. Let $S=\left\{x_{1}, x_{2}, x_{3}, \ldots, x_{n}\right\}$ be a subset of $P$ and the $n \times n$ matrix $(S)_{f}=\left(f_{i j}\right)$ where

$$
f_{i j}=2^{2^{4\left(x_{i} \wedge x_{j}\right)+1}}+1 \text {, is called the Fermat S-prime Meet matrix on S. }
$$


The Fermat S-Prime Meet Matrices and Reciprocal Fermat S-Prime Meet Matrices on Posets

Definition 2.8. Let $S=\left\{x_{1}, x_{2}, x_{3}, \ldots, x_{n}\right\}$ be an ordered set of distinct positive integer. The Reciprocal Fermat S-prime Meet matrix on $S$ is defined as $(S)_{1 / f}=\left(f_{i j}\right)$ where

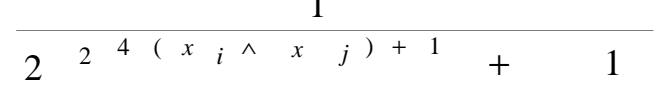

Definition 2.9. Let $S=\left\{x_{1}, x_{2}, x_{3}, \ldots, x_{n}\right\}$ be a subset of $P$, and let $f$ be a function on $P$ with complex values. Then the function $g_{s, f}$ on $\mathrm{S}$ is defined inductively by

$$
g_{s, f}\left(x_{j}\right)=f\left(x_{j}\right)-\sum_{x_{i} \leq x_{j}} g_{s, f}\left(x_{i}\right)
$$

where $\mathrm{x}_{\mathrm{i}}<\mathrm{x}_{\mathrm{j}}$ means that $\mathrm{x}_{\mathrm{i}} \neq \mathrm{x}_{\mathrm{j}}$ or $f\left(x_{j}\right)=\sum_{x_{i} \leq x_{j}} g_{s, f}\left(x_{i}\right) \quad(p .2,[8])$

3. Determinant and inverse of the fermat $S$-prime meet matrices on posets

Theorem 3.1. Let $S=\left\{x_{1}, x_{2}, x_{3}, \ldots, x_{n}\right\}$ be S-prime Meet-closed . Without loss of generality we may assume that $\mathrm{i}<\mathrm{j}$ whenever $\mathrm{x}_{\mathrm{i}}<\mathrm{x}_{\mathrm{j}}$, then

$$
g_{s, f}\left(x_{j}\right)=\sum_{z \leq x_{j}} \sum_{\substack{w \leq z \\ z \leq x_{t} \\ t<j}} f(w) \mu(w, z) \text { where } \mu \text { is the mobius function of } \mathrm{P} \text {. }
$$

Proof: By using the definition (2.9),

$$
f\left(x_{j}\right)=\sum_{x_{i} \leq x_{j}} g_{s, f}\left(x_{i}\right)=\sum_{x_{i} \leq x_{j}} \sum_{\substack{z \leq x_{j} \\ z<x_{i} \leq z \\ i<j}} f(w) \mu(w, z)
$$

We write, $f(x)=\sum_{z \leq x} g_{s, f}(z)$ or $g_{s, f}(x)=\sum_{z \leq x} f(z) \mu(z, x) \quad$ for all $\mathrm{x} \in \mathrm{P}$

$$
\text { It has to be prove that } \sum_{z \leq x_{j}} g_{s, f}(z)=\sum_{x_{i} \leq x_{j}} \sum_{\substack{z \leq x_{i} \\ z \leq x_{i} \\ t<i}} g_{s, f}(z)
$$

Now consider the sum of R.H.S of equation (1)

Let $\mathrm{x}_{\mathrm{i}} \leq \mathrm{x}_{\mathrm{j}}$ and $\mathrm{z} \leq \mathrm{x}_{\mathrm{i}} \Rightarrow \mathrm{z} \leq \mathrm{x}_{\mathrm{j}}$. Thus every $\mathrm{z}$ occurring on the right side of equation (1) occurs on the left side of equation (1).

Conversely, consider the sum on the left side of equation (1).

Suppose that $\mathrm{z} \leq \mathrm{x}_{\mathrm{j}}$ we have $\mathrm{z} \leq \mathrm{x}_{\mathrm{i}}$ by minimality of $\mathrm{i}$, we have $\mathrm{r}=\mathrm{i}$ or $\mathrm{x}_{\mathrm{r}}=\mathrm{x}_{\mathrm{i}}$, therefore $\mathrm{x}_{\mathrm{r}}$ $\leq x_{j}$ means $\quad x_{r} \leq x_{j}$ thus every $z$ occurring on the side of equation (1).

This completes the proof of the theorem.

Theorem 3.2. If $\mathrm{S}$ is lower closed subset of $\mathrm{P}$. Then

$$
g_{s, f\left(x_{j}\right)}=\sum_{x_{i} \leq x_{j}} f\left(x_{i}\right) \mu\left(x_{i}, x_{j}\right)
$$

Proof: Already we know that the result,

$$
g_{s, f\left(x_{j}\right)}=\sum_{\substack{z \leq x_{j} \\ z \leq x_{t} \\ t<j}} f(w) \mu(w, z)
$$

It reduces we get the proof of theorem [11]. Then S is lower closed. 
N.Elumalai and R.Anuradha

Example 3.3. Let $\mathrm{S}=\left\{\mathrm{x}_{1}, \mathrm{x}_{2}, \ldots \ldots . . \mathrm{x}_{\mathrm{n}}\right\}$ be a chain with $\mathrm{x}_{1}<\mathrm{x}_{2}<\ldots \ldots<\mathrm{x}_{\mathrm{n}}$. Then $\mathrm{g}_{\mathrm{s}, \mathrm{f}}\left(\mathrm{x}_{1}\right)=\mathrm{f}\left(\mathrm{x}_{1}\right)$, $\mathrm{g}_{\mathrm{s}, \mathrm{f}}\left(\mathrm{x}_{2}\right)=\mathrm{f}\left(\mathrm{x}_{2}\right)-\mathrm{f}\left(\mathrm{x}_{1}\right)$. In general $g_{s, f}\left(x_{j}\right)=f\left(x_{j}\right)-f\left(x_{j-1}\right)$ where, $\mathrm{j}=2,3,4, \ldots, \mathrm{n}$.

Example 3.4. Let $\mathrm{S}=\left\{\mathrm{x}_{1}, \mathrm{x}_{2}, \ldots . . \mathrm{x}_{\mathrm{n}}\right\}$ be an incomparable set and let $\mathrm{S}=\left\{\mathrm{x}_{0}, \mathrm{x}_{1}, \mathrm{x}_{2}\right.$ ,$\left.\ldots . . \mathrm{x}_{\mathrm{n}}\right\}$. Then, $g_{s, f}\left(x_{0}\right)=f\left(x_{0}\right), g_{s, f}\left(x_{1}\right)=f\left(x_{1}\right)-f\left(x_{0}\right)$, and $g_{s, f}\left(x_{2}\right)=f\left(x_{2}\right)-f\left(x_{0}\right)$. In general $g_{s, f}\left(x_{j}\right)=f\left(x_{j}\right)-f\left(x_{0}\right)$ for $\mathrm{j}=1,2,3, \ldots, \mathrm{n}$

Theorem 3.5. Let $S=\left\{x_{1}, x_{2}, x_{3}, \ldots, x_{n}\right\}$ be a meet - closed subset of $P$ then $\operatorname{det}(\mathrm{S})_{\mathrm{f}}=\mathrm{g}_{\mathrm{s}, \mathrm{f}}\left(\mathrm{x}_{1}\right) \mathrm{g}_{\mathrm{s}, \mathrm{f}}\left(\mathrm{x}_{2}\right) \ldots \mathrm{g}_{\mathrm{s}, \mathrm{f}}\left(\mathrm{x}_{\mathrm{n}}\right)$ where $\mathrm{g}_{\mathrm{s}, \mathrm{f}}\left(\mathrm{x}_{\mathrm{i}}\right)$ defined by $g_{s, f}\left(x_{i}\right)=\left(2^{2^{4_{x_{i}+1}}}+1\right)-\sum_{x_{j} \in S, x_{j} \prec x_{i}} g_{s, f}\left(x_{j}\right) \cdot[11]$

Theorem 3.6. Let $S=\left\{x_{1}, x_{2}, x_{3}, \ldots, x_{n}\right\}$ be a lower - closed subset of $P$ then $\operatorname{det}(\mathrm{S})_{\mathrm{f}}=\mathrm{g}_{\mathrm{s}, \mathrm{f}}\left(\mathrm{x}_{1}\right) \mathrm{g}_{\mathrm{s}, \mathrm{f}}\left(\mathrm{x}_{2}\right) \ldots \mathrm{g}_{\mathrm{s}, \mathrm{f}}\left(\mathrm{x}_{\mathrm{n}}\right)$ where $g_{s, f}\left(x_{i}\right)=\sum_{x_{i \wedge} \wedge x_{i}}\left(2^{2^{4 x_{i}+1}}+1\right) \mu\left(x_{j}, x_{i}\right)$

Proof: The theorem is proved and verified with a suitable example.

Consider the set $S=\{1,2\}$

By using the definition (2.7), we have

$$
\begin{aligned}
& (\mathrm{S})_{\mathrm{f}}=\left(\begin{array}{ll}
2^{2^{4(1 \wedge 1)+1}+1} & 2^{2^{4(1 \wedge 2)+1}+1} \\
2^{2^{4(2 \wedge 1)+1}+1} & 2^{2^{4(2 \wedge 2)+1}+1}
\end{array}\right)=\left(\begin{array}{ll}
2^{32}+1 & 2^{32}+1 \\
2^{32}+1 & 2^{512}+1
\end{array}\right) \\
& \text { since } g_{s, f}\left(x_{i}\right)=\sum_{x_{s \wedge} x_{i}}\left(2^{2^{4 x_{i}+1}}+1\right) \mu\left(x_{j}, x_{i}\right)
\end{aligned}
$$

Theorem 3.7. Let $S=\left\{x_{1}, x_{2}, x_{3}, \ldots, x_{n}\right\}$ and define $m \times m$ matrix

$$
\Lambda=\operatorname{diag}\left(g\left(d_{1}\right), g\left(d_{2}\right), \ldots, g\left(d_{m}\right)\right) \text { where } g(n)=\sum_{d / n}\left(2^{2^{4 d+1}}+1\right) \mu\left(\frac{n}{d}\right) \text { and } n \times m
$$

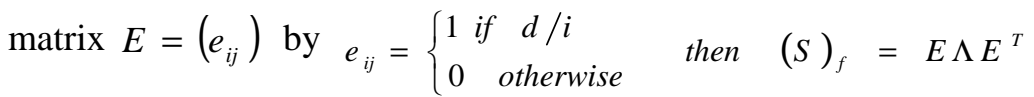

Proof: The ij- entry in $E \Lambda E^{T}$ is

$$
\left(E \Lambda E^{T}\right)_{i j}=\sum_{k=1}^{n} e_{i k} \Lambda_{k} e_{k j}=\sum_{\substack{d_{k} / x_{i} \\ d_{k} / x_{j}}} g\left(d_{k}\right)=\sum_{d_{k} / x_{i} \wedge x_{j}} g\left(d_{k}\right)=2^{2^{4 d+1}}+1=f_{i j}
$$


The Fermat S-Prime Meet Matrices and Reciprocal Fermat S-Prime Meet Matrices on Posets

Theorem 3.8. Let $\mathrm{S}=\left\{\mathrm{x}_{1}, \mathrm{x}_{2}, \mathrm{x}_{3}, \ldots, \mathrm{x}_{\mathrm{n}}\right\}$ be a subset of $\mathrm{P}$ with $\bar{S}$ $=\left\{\mathrm{x}_{1}, \mathrm{x}_{2}, \ldots, \mathrm{x}_{\mathrm{n}}, \mathrm{x}_{\mathrm{n}+1}, \ldots, \mathrm{x}_{\mathrm{n}+\mathrm{r}}\right\}$. Let $\mathrm{g}$ be a function on $\bar{S}$ defined as in theorem(3.5).Then $(\mathrm{S})_{\mathrm{f}}=\mathrm{E} \Lambda \mathrm{E}^{\mathrm{T}}$ and $\mathrm{E}^{\mathrm{T}}$ is the transpose of $\mathrm{E}$.

Proof: The theorem is proved and verified with a suitable example.

Consider the set $\mathrm{S}=\{1,2\}, \bar{S}=\{1,2,3\}$

By using the definition (2.6), we have

$$
(S)_{f}=f\left(2^{2^{4\left(x_{i} \wedge x_{j}\right)+1}}+1\right)=\left(\begin{array}{ll}
f\left(2^{2^{5}}+1\right) & f\left(2^{2^{5}}+1\right) \\
f\left(2^{2^{5}}+1\right) & f\left(2^{2^{9}}+1\right)
\end{array}\right)=\left(\begin{array}{ll}
f\left(2^{32}+1\right) & f\left(2^{32}+1\right) \\
f\left(2^{32}+1\right) & f\left(2^{512}+1\right)
\end{array}\right)
$$

since $g_{s, f}\left(x_{j}\right)=f\left(x_{j}\right)-f\left(x_{j-1}\right)$ where $j=1,2, \ldots, n$,

$$
\begin{aligned}
& \therefore g_{s, f}\left(x_{1}\right)=f\left(x_{1}\right)=f(1)=f\left(2^{2^{5}}+1\right)=f\left(2^{32}+1\right) \\
& g_{s, f}\left(x_{2}\right)=f\left(x_{2}\right)-f\left(x_{1}\right)=\left(2^{512}+1\right)-f\left(2^{32}+1\right), g_{s, f}\left(x_{3}\right)=f\left(2^{8192}+1\right)-f\left(2^{512}+1\right) \\
& \Lambda=\operatorname{diag}\left(g_{s, f}\left(x_{1}\right), g_{s, f}\left(x_{2}\right), g_{s, f}\left(x_{3}\right)\right)\left(\begin{array}{ccc}
f\left(2^{32}+1\right) & 0 & 0 \\
0 & f\left(2^{512}+1\right)-f\left(2^{32}+1\right) & 0 \\
0 & 0 & f\left(2^{8192}+1\right)-f\left(2^{512}+1\right)
\end{array}\right)
\end{aligned}
$$

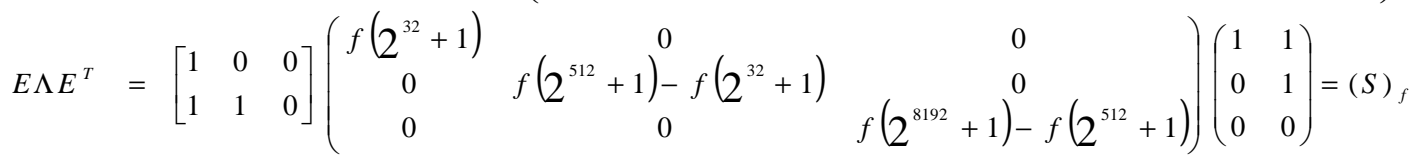

Theorem 3.9. Let $T=\left\{y_{1}, y_{2}, y_{3}, \ldots, y_{m}\right\}$ be a S-prime Meet -closed subset of $P$ containing $\mathrm{S}=\left\{\mathrm{x}_{1}, \mathrm{x}_{2}, \mathrm{x}_{3, \ldots, \ldots} \mathrm{x}_{\mathrm{n}}\right\}$. Then,

$$
\operatorname{det}(\mathbf{S})_{\mathrm{f}}=\sum_{1 \leq k_{1} \leq \ldots \leq k_{n} \leq m} \operatorname{det}\left[E\left(k_{1}, k_{2}, \ldots, k_{n}\right)^{2}\right] g_{T, f}(y)_{k_{1}}, g_{T, f}(y)_{k_{2}}, \ldots g_{T, f}(y)_{k_{n}}
$$

where, $\mathrm{E}=\mathrm{E}(\mathrm{S}, \mathrm{T})$ is the submatrix of $\mathrm{E}=\mathrm{E}(\mathrm{S}, \bar{S})$ consisting of the $\mathrm{k}_{1}$ th, $\mathrm{k}_{2}$ th $, \ldots, \mathrm{k}_{\mathrm{n}}$ th columns of $\mathrm{E}$.

Proof: Since $(S)_{f}=E \Lambda E^{T}$ and also $\operatorname{det}(E)=\operatorname{det}\left(E^{T}\right)$, by using Cauchy Binet Formula[10] to get the proof of the theorem.

Example 3.10. Let $S=\{1,3\}$

By using the definition(2.7), we have

$$
\begin{gathered}
(\mathrm{S})_{\mathrm{f}}=\left(\begin{array}{cc}
2^{2^{4(1 \wedge 1)+1}+1} & 2^{2^{4(1 \wedge 3)+1}+1} \\
2^{2^{4(3 \wedge 1)+1}}+1 & 2^{2^{4(3 \wedge 3)+1}}+1
\end{array}\right)=\left(\begin{array}{cc}
2^{32}+1 & 2^{32}+1 \\
2^{32}+1 & 2^{8192}+1
\end{array}\right) \\
\therefore \operatorname{det}(S)_{f}=\left(2^{32}+1\right)\left(2^{8192}+1\right)-\left(2^{32}+1\right)^{2} \\
\text { since } g_{g_{T, f}}\left(x_{i}\right)=\sum_{x_{j \wedge} x_{i}}\left(2^{2^{4 x_{i}+1}}+1\right) \mu\left(x_{j}, x_{i}\right),
\end{gathered}
$$


N.Elumalai and R.Anuradha

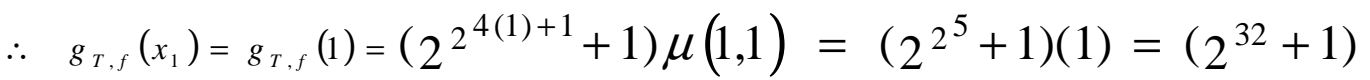

$$
\begin{aligned}
& g_{T, f}\left(x_{2}\right)=g_{T, f}(3)=\left(2^{2^{5}}+1\right)(-1)+\left(2^{2^{13}}+1\right)(1)=-\left(2^{32}+1\right)+\left(2^{8192}+1\right) \\
& \sum_{1 \leq k_{1} \leq \ldots \leq k_{n} \leq m} \operatorname{det}\left[E\left(k_{1}, k_{2}, \ldots, k_{n}\right)^{2}\right] g_{T, f}(y)_{k 1}, g_{T, f}(y)_{k 2}, \ldots g_{T, f}(y)_{k n} \\
& =\left|\begin{array}{ll}
1 & 0 \\
1 & 1
\end{array}\right|^{2} g_{s, f}\left(x_{1}\right) g_{s, f}\left(x_{2}\right)+\left|\begin{array}{ll}
1 & 0 \\
1 & 0
\end{array}\right|^{2} g_{s, f}\left(x_{1}\right) g_{s, f}\left(x_{3}\right)+\left|\begin{array}{ll}
0 & 0 \\
1 & 0
\end{array}\right|^{2} g_{s, f}\left(x_{2}\right) g_{s, f}\left(x_{3}\right) \\
& =g_{s, f}\left(x_{1}\right) g_{s, f}\left(x_{2}\right)=\left(2^{32}+1\right)\left[-\left(2^{32}+1\right)+\left(2^{8192}+1\right)\right]
\end{aligned}
$$

Hence $\operatorname{det}(\mathrm{S})_{\mathrm{f}}=\sum_{1 \leq k_{1} \leq \ldots \leq k_{n} \leq m} \operatorname{det}\left[E\left(k_{1}, k_{2}, \ldots, k_{n}\right)^{2}\right] g_{T, f}(y)_{k 1}, g_{T, f}(y)_{k 2}, \ldots . g_{T, f}(y)_{k n}$

Theorem 3.11. Let $S=\left\{x_{1}, x_{2}, x_{3}, \ldots, x_{n}\right\}$ be a lower closed subset of $P$ and let

$$
g_{s, f}\left(x_{i}\right)=\sum_{x, \wedge x_{i}}\left(2^{2 x^{4} x_{i}+1}+1\right) \mu\left(x_{j}, x_{i}\right) \neq 0 \text { for all } x_{j} \in S
$$

Then $(S)_{\mathrm{f}}$ isinvertibleand $\left(\mathrm{S}_{\mathrm{f}}^{-1}=\left(\mathrm{c}_{\mathrm{ij}}\right)\right.$,

where $\mathrm{c}_{\mathrm{ij}}=\sum_{\substack{x_{i} \leq x_{k} \\ x_{j} \leq x_{k}}} \frac{1}{g_{s, f}\left(x_{k}\right)} \mu\left(x_{i}, x_{k}\right) \mu\left(x_{j}, x_{k}\right)$

\section{Proof:}

The $n \times n$ matrix $[Y]=\left(y_{i j}\right)$ defined by $y_{i j}=\left\{\begin{array}{l}\mu\left(x_{i}, x_{j}\right) ; x_{i} x_{j} \\ 0 \quad ; \text { otherwise }\end{array}\right.$

Calculating the ij-entry of product EY gives,

$$
(E Y)_{i j}=\sum_{k=1}^{n} e_{i k} y_{k j}=\sum_{\substack{x_{k} / x_{j} \\ x_{j} / x_{k}}} \mu\left(x_{k}, x_{j}\right)=\sum_{x_{k} / \frac{x_{i}}{x_{j}}} \mu\left(x_{k}\right)= \begin{cases}1 & \text { if } i=j \\ 0 & \text { otherwise }\end{cases}
$$

Thus $\mathrm{E}^{-1}=\mathrm{Y}$.

$(\mathrm{S})_{\mathrm{f}}=\mathrm{E} \Lambda \mathrm{E}^{\mathrm{T}}$ and $\mathrm{E}^{-1}=\mathrm{Y}$ then $(S)_{f}^{-1}=\left(\mathrm{E} \Lambda \mathrm{E}^{\mathrm{T}}\right)^{-1}=Y^{T} \Lambda^{1 / 2} Y=c_{i j}$

Thus, the proof is complete.

\section{Example 3.12.}

Let $S=\{1,3\}$ be a lower closed subset of $P$ then, $(S)_{f}{ }^{-1}=\left(c_{i j}\right)$ where $c_{11}=\frac{1}{g_{s, f}(1)} \mu(1,1) \mu(1,1)=\frac{1}{\left(2^{32}+1\right)} \mu(1,1)^{2}=\frac{1}{2^{32}+1}$ 
The Fermat S-Prime Meet Matrices and Reciprocal Fermat S-Prime Meet Matrices on Posets

$$
\begin{aligned}
& c_{12}=\sum_{\substack{1 \leq x_{k} \\
2 \leq x_{k}}} \frac{1}{g_{s, f}\left(x_{k}\right)} \mu\left(1, x_{k}\right) \mu\left(3, x_{k}\right)=\frac{\mu(1,3) \mu(3,3)}{g_{s, f}(3)}=\frac{(-1)(1)}{-\left(2^{32}+1\right)+\left(2^{8192}+1\right)} \\
& \text { similarly }, c_{21}=\frac{(-1)(1)}{-\left(2^{32}+1\right)+\left(2^{8192}+1\right)}, c_{22}= \\
& \therefore \quad(S)_{f}^{-1}=\left(c_{i j}\right)=\left(\begin{array}{cc}
\frac{1}{2^{32}+1} & \frac{1}{-\left(2^{32}+1\right)+\left(2^{8192}+1\right)} \\
\frac{-1}{-\left(2^{32}+1\right)+\left(2^{8192}+1\right)} & \frac{-1}{-\left(2^{32}+1\right)+\left(2^{8192}+1\right)}
\end{array}\right)
\end{aligned}
$$

\section{Determinant and inverse of the reciprocal fermat S-prime meet matrices on} posets

Theorem 4.1. Let $S=\left\{x_{1}, x_{2}, x_{3}, \ldots, x_{n}\right\}$ be a meet - closed subset of $P$ then $\operatorname{det}(S)_{1 / f}=$ $\mathrm{g}_{\mathrm{s}, 1 / \mathrm{f}}\left(\mathrm{x}_{1}\right) \mathrm{g}_{\mathrm{s}, 1 / \mathrm{f}}\left(\mathrm{x}_{2}\right) \ldots \mathrm{g}_{\mathrm{s}, 1 / \mathrm{f}}\left(\mathrm{x}_{\mathrm{n}}\right)$ where $\mathrm{g}_{\mathrm{s}, 1 / \mathrm{f}}\left(\mathrm{x}_{\mathrm{i}}\right)$ defined by $g_{s, 1 / f}\left(x_{i}\right)=\left(\frac{1}{2^{2^{x_{i}+1}}+1}\right) \mu\left(x_{i}, x_{j}\right)$

Proof: The theorem is proved and verified with a suitable example.

Consider the set $S=\{1,2\}$

By using the definition(2.8), we have

$$
\begin{aligned}
& (\mathrm{S})_{1 / \mathrm{f}}=\left(\begin{array}{cc}
\frac{1}{2^{2^{4(1 \wedge 1)+1}+1}} & \frac{1}{2^{2^{4(1 \wedge 2)+1}+1}} \\
\frac{1}{2^{2^{4(2 \wedge 1)+1}+1}} & \frac{1}{2^{2^{4(2 \wedge 2)+1}+1}}
\end{array}\right)=\left(\begin{array}{cc}
\frac{1}{2^{32}+1} & \frac{1}{2^{32}+1} \\
\frac{1}{2^{32}+1} & \frac{1}{2^{512}+1}
\end{array}\right) \\
& \therefore \quad g_{s, 1 / f}\left(x_{1}\right)=g_{s, 1 / f}(1)=\left(\frac{1}{2^{2^{4(1)+1}+1}}\right) \mu(1,1)=\left(\frac{1}{2^{2^{5}+1}}\right)(1)=\frac{1}{2^{32}+1} \\
& g_{s, 1 / f}\left(x_{2}\right)=g_{s, 1 / f}(2)=\left(\frac{1}{2^{2^{5}+1}}\right)(-1)+\left(\frac{1}{2^{2^{9}+1}}\right)(1)=\left(\frac{-1}{2^{32}+1}\right)+\left(\frac{1}{2^{512}+1}\right) \\
& \text { Now } \quad g_{s, 1 / f}\left(x_{1}\right) g_{s, 1 / f}\left(x_{2}\right)=g_{s, 1 / f}(1) \cdot g_{s, 1 / f}(2)=\left(\frac{1}{2^{32}+1}\right)\left[\left(\frac{-1}{2^{32}+1}\right)+\left(\frac{1}{2^{512}+1}\right)\right]
\end{aligned}
$$

Hence $\operatorname{det}(\mathrm{S})_{1 / \mathrm{f}}=\mathrm{g}_{\mathrm{s}, 1 / \mathrm{f}}\left(\mathrm{x}_{1}\right) \mathrm{g}_{\mathrm{s}, 1 / \mathrm{f}}\left(\mathrm{x}_{2}\right)$.

Theorem 4.2. Let $\mathrm{S}=\left\{\mathrm{x}_{1}, \mathrm{x}_{2}, \mathrm{x}_{3}, \ldots, \mathrm{x}_{\mathrm{n}}\right\}$ be a subset of $\mathrm{P}$ with $\bar{S}$ $=\left\{\mathrm{x}_{1}, \mathrm{x}_{2}, \ldots, \mathrm{x}_{\mathrm{n}}, \mathrm{x}_{\mathrm{n}+1}, \ldots, \mathrm{x}_{\mathrm{n}+\mathrm{r}}\right\}$. Let $\mathrm{g}$ be a function on $\bar{S}$ defined as in theorem(4.1).Then $(\mathrm{S})_{1 / \mathrm{f}}=\mathrm{E} \wedge \mathrm{E}^{\mathrm{T}}$ and $\mathrm{E}^{\mathrm{T}}$ is the transpose of $\mathrm{E}$.

Proof: The theorem is proved and verified with a suitable example.

Consider the set $\mathrm{S}=\{1,3\}, \bar{S}=\{1,3,5\}$ 


\section{N.Elumalai and R.Anuradha}

By using the definition(2.8), we have

$$
(\mathrm{S})_{1 / \mathrm{f}}=f\left(\frac{1}{2^{2^{4\left(x_{i} \wedge x_{j}\right)+1}}+1}\right)=\left(\begin{array}{cc}
f\left(\frac{1}{2^{32}+1}\right) & f\left(\frac{1}{2^{32}+1}\right) \\
f\left(\frac{1}{2^{32}+1}\right) & f\left(\frac{1}{2^{8192}+1}\right)
\end{array}\right)
$$

since $g_{s, 1 / f}\left(x_{j}\right)=f\left(x_{j}\right)-f\left(x_{j-1}\right)$ where $j=1,2, \ldots, n$

$\therefore g_{s, 1 / f}\left(x_{1}\right)=f\left(x_{1}\right)=f(1)=f\left(\frac{1}{2^{2^{5}+1}}\right)=f\left(\frac{1}{2^{32}+1}\right)$$$
g_{s, 1 / f}\left(x_{2}\right)=f\left(\frac{1}{2^{8192}+1}\right)-f\left(\frac{1}{2^{32}+1}\right), g_{s, 1 / f}\left(x_{3}\right)=f\left(\frac{1}{2^{2^{21}}+1}\right)-f\left(\frac{1}{2^{2^{13}}+1}\right)
$$$$
f\left(\frac{1}{2^{32}+1}\right) \quad 0 \quad 0
$$$$
\therefore \Lambda=\left(\begin{array}{ccc}
0 & f\left(\frac{1}{2^{8192}+1}\right)-f\left(\frac{1}{2^{32}+1}\right) & 0 \\
0 & 0 & f\left(\frac{1}{2^{2^{21}}+1}\right)-f\left(\frac{1}{2^{2^{13}}+1}\right)
\end{array}\right)
$$$$
E \Lambda E^{T}=\left[\begin{array}{lll}
1 & 0 & 0 \\
1 & 1 & 0
\end{array}\right]\left(\begin{array}{ccc}
f\left(\frac{1}{2^{32}+1}\right) & 0 & 0 \\
0 & f\left(\frac{1}{2^{8192}+1}\right)-f\left(\frac{1}{2^{32}+1}\right) & 0 \\
0 & 0 & f\left(\frac{1}{2^{2^{21}}+1}\right)-f\left(\frac{1}{2^{2^{13}}+1}\right)
\end{array}\right)\left(\begin{array}{ll}
1 & 1 \\
0 & 1 \\
0 & 0
\end{array}\right)=(S)_{f}
$$

\section{Theorem 4.3.}

Let $\mathrm{T}=\left\{\mathrm{y}_{1}, \mathrm{y}_{2}, \mathrm{y}_{3}, \ldots, \mathrm{y}_{\mathrm{m}}\right\}$ be a S-prime Meet -closed subset of P containing $\mathrm{S}=\left\{\mathrm{x}_{1}, \mathrm{x}_{2}, \mathrm{x}_{3, \ldots}, \ldots, \mathrm{x}_{\mathrm{n}}\right\}$. Then,

$\operatorname{det}(\mathrm{S})_{1 / \mathrm{f}}=\sum_{1 \leq k_{1} \leq \ldots \leq k_{n} \leq m}^{\operatorname{det}}\left[E\left(k_{1}, k_{2}, \ldots, k_{n}\right)^{2}\right] g_{T, 1 / f}(y)_{k_{1}}, g_{T, 1 / f}(y)_{k_{2}}, \ldots . g_{T, 1 / f}(y)_{k_{n}}$ where,

$\mathrm{E}=\mathrm{E}(\mathrm{S}, \mathrm{T})$ is the submatrix of $\mathrm{E}=\mathrm{E}(\mathrm{S}, \bar{S})$ consisting of the $\mathrm{k}_{1}$ th $, \mathrm{k}_{2} \mathrm{th}, \ldots, \mathrm{k}_{\mathrm{n}}$ th columns of E.

Proof: The theorem is proved and verified with a suitable example. Let $\mathrm{S}=\{1,5\}$

By using the definition(2.8), we have 
The Fermat S-Prime Meet Matrices and Reciprocal Fermat S-Prime Meet Matrices on Posets

$$
\begin{aligned}
& (\mathrm{S})_{1 / \mathrm{f}}=\left(\begin{array}{cc}
\frac{1}{2^{2^{4(1 \wedge 1)+1}+1}} & \frac{1}{2^{2^{4(1 \wedge 5)+1}+1}} \\
\frac{1}{2^{2^{4(5 \wedge 1)+1}+1}} & \frac{1}{2^{2^{4(5 \wedge 5)+1}+1}}
\end{array}\right)=\left(\begin{array}{cc}
\frac{1}{2^{32}+1} & \frac{1}{2^{32}+1} \\
\frac{1}{2^{32}+1} & \frac{1}{2^{2^{21}}+1}
\end{array}\right) \\
& \operatorname{det}(S)_{1 / f}=\left(\frac{1}{2^{32}+1}\right)\left(\frac{1}{2^{21}+1}\right)-\left(\frac{1}{2^{32}+1}\right)^{2} \\
& \text { since } g_{s, 1, f}\left(x_{i}\right)=\sum_{x_{j} \wedge x_{i}}\left(\frac{1}{2^{24 x_{i}+1}+1}\right) \mu\left(x_{j}, x_{i}\right) \\
& \therefore \quad g_{s, 1 / f}\left(x_{1}\right)=g_{s, 1 / f}(1)=\left(\frac{1}{2^{2^{4(1)+1}+1}}\right) \mu(1,1)=\left(\frac{1}{2^{2^{5}+1}}\right)(1)=\left(\frac{1}{2^{32}+1}\right) \\
& g_{s, 1 / f}\left(x_{2}\right)=g_{s, 1 / f}(5)=\left(\frac{1}{2^{2^{5}+1}}\right)(-1)+\left(\frac{1}{2^{2^{21}}+1}\right)(1)=\left(\frac{-1}{2^{32}+1}\right)+\left(\frac{1}{2^{2^{21}}+1}\right)
\end{aligned}
$$

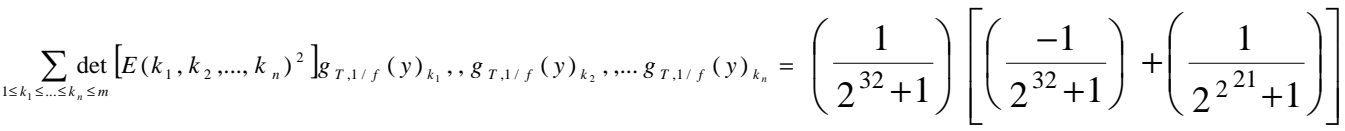

Hence $\operatorname{det}(\mathrm{S})_{1 / \mathrm{f}}=\sum_{1 \leq k_{1} \leq \ldots \leq k_{n} \leq m} \operatorname{det}\left[E\left(k_{1}, k_{2}, \ldots, k_{n}\right)^{2}\right] g_{T, 1 / f}(y)_{k_{1}},, g_{T, 1 / f}(y)_{k_{2}}, \ldots . g_{T, 1 / f}(y)_{k_{n}}$

Theorem 4.4. Let $S=\left\{x_{1}, x_{2}, x_{3}, \ldots, x_{n}\right\}$ be a lower closed subset of $P$ and let $g_{s, 1 / f}\left(x_{i}\right)=\sum_{x_{i} \wedge x_{i}}\left(\frac{1}{2^{2^{4 x_{i}+1}+1}}\right) \mu\left(x_{j}, x_{i}\right) \neq 0$ for all $x_{j} \in S$.

Then $(\mathrm{S})_{1 / f}$ is invertible and $\left(\mathrm{S}_{1 / \mathrm{f}}^{-1}=\left(\mathrm{d}_{\mathrm{ij}}\right)\right.$, where

$d_{i j}=\sum_{\substack{x_{i} \leq x_{k} \\ x_{j} \leq x_{k}}} \frac{1}{g_{s, 1 / f}\left(x_{k}\right)} \mu\left(x_{i}, x_{k}\right) \mu\left(x_{j}, x_{k}\right)$.[6]

Example 4.5. Let $S=\{1,5\}$ be a lower closed subset of $P$ then $(S)_{1 / f}^{-1}=\left(d_{i j}\right)$ where

$$
d_{11}=2^{32}+1, d_{12}=\frac{-1}{\left(\frac{-1}{2^{32}+1}\right)+\left(\frac{1}{2^{221}+1}\right)}
$$


N.Elumalai and R.Anuradha

$$
\begin{aligned}
& d_{21}=\frac{-1}{\left(\frac{-1}{2^{32}+1}\right)+\left(\frac{1}{2^{2}+1}\right)}, d_{22}=\frac{1}{\left(\frac{-1}{2^{32}+1}\right)+\left(\frac{1}{2^{2}+1}\right)} \\
& \therefore \quad(S)_{1, f^{-1}}=\left(d_{i j}\right)=\left(\frac{2^{32}+1}{\frac{-1}{\left(\frac{-1}{2^{32}+1}\right)+\left(\frac{1}{2^{21}+1}\right)}} \frac{-1}{\left(\frac{-1}{2^{32}+1}\right)+\left(\frac{1}{2^{22}+1}\right)+\left(\frac{1}{2^{22}+1}\right)}\right)
\end{aligned}
$$

\section{REFERENCES}

1. E.Altinisik, N.Tuglu and P.Haukkanen, Determinant and inverse of meet and join matrices, International journal of Mathematics and Mathematical Sciences, (2007) E1-11.

2. T.M.Apostal, Introdution to Analytical Number Theory, Springer International Student Edition, (1980).

3. G.P.Berger, The determinant of GCD matrices, Linear Algebra and its Application, 134 (1990) 137-143.

4. S. Beslin and S.Ligh, Greatest common disivor matrices, Linear Algebra Appl., 118 (1989) 69-76.

5. S.Beslin, Reciprocal GCD matrices and LCM matrices, Fibonacci Quarterly Journal 29 (1991) 271-274.

6. K.Bourque and S.Ligh, On GCD and LCM matrices, Linear Algebra and Its Appl., 174 (1992) 65-74.

7. S.Buyukkose, The fermat meet and reciprocal's fermat meet matrices on posets, International Journal of Contemporary Mathematical Sciences, 3(35) (2008) 1739 1744.

8. S.Buyukkose and D.Tasci, The fermat GCD matrices, International Journal of Contemporary Mathematical Sciences, 3(35) 1745-1753.

9. L.Carlitz, Some matrices related to the greatest integer function, J. Elisha Mitchell Sci. Soc., 76 (1960) 5-7.

10. N.Elumalai, R.Anuradha and S.Praveena, S-prime meet matrices on posets, International Journal of Scientific, Engineering and Technology Research, 5 (2016) 420-425.

11. H.J.S. Smith, On the value of a certain arithmetical determinant, Proc. London Math. Soc., 7 (1875) 208-213. 